Medicaciones antiobesidad como topiramato/fentermina, lorcaserina, naltrexona/bupropión, orlistat y fentermina son efectivas pero no existen estudios realizados específicamente en pacientes con DM1.

En cuanto a la cirugía metabólica ambas técnicas, by pass en Y de Roux y manga gástrica, se implementaron en pacientes con DM1 con resultados discutibles.

Es necesario generar guías de estrategias para prevenir y controlar la ganancia de peso en pacientes con DM1 que requieran tratamiento insulínico intensificado.

\title{
BIBLIOGRAFÍA
}

- Corbin KD, Driscoll KA, Pratley RE, Smith SR, Maahs DM, Mayer-Davis EJ; Advancing Care forType 1 Diabetes and Obesity Network (ACT1ON). Obesity in type 1 diabetes: pathophysiology, clinical impact, and mechanisms. Endocrine Reviews 2018; 39: 629-663.

- Polskya S, Ellisb SL. Obesity, insulin resistance, and type 1 diabetes mellitus. Curr Opin Endocrinol Diabetes Obes 2015; 22:277-282.

- Mottalib A, Kasetty M, Mar JY, ElseaidyT, Ashrafzadeh S, Hamdy O. Weight management in patients with type 1 diabetes and obesity. Curr Diab Rep 2017 Aug 23; 17(10):92. Doi: 10.1007/s11892-017-0918-8.

\section{Enfermedad celíaca y diabetes mellitus tipo 1}

Dra. Mabel Ferraro

La joven nieta de Juana (21 años) es portadora de diabetes mellitus tipo 1 (DM1), obesidad y enfermedad celíaca $(E C)$. La EC se origina por la reacción inmune mediada por células $T$ al gluten de la dieta y se caracteriza por la inflamación del intestino delgado, atrofia vellosa, hiperplasia de la cripta y malabsorción. La prevalencia de esta asociación es del $1 \%$ a nivel mundial, muy variable en los diferentes países y asciende del 4 al 10\% si se asocia con DM11. La base genética de la misma es un HLA característico DQ2/DQ8 necesario pero no excluyente para su aparición. Se conocen otros genes no HLA de susceptibilidad como CTLA-4, PTPN22, VDR, IL-2Ra y TNF- $\alpha$ que pudiera presentar esta paciente ${ }^{2}$.

En los últimos años se ha revalorizado el papel de la microbiota como protectora o favorecedora de su aparición. Sería interesante recabar si la joven nació por vía vaginal, recibió lactancia materna y el momento de incorporación del gluten. La presencia de bifidobacterias y lactobacilos podría haber disminuido el riesgo ${ }^{3}$. El diagnóstico debió realizarse por pesquisa sistemática indispensable en los portadores de DM1. Según la International Society for Pediatric and Adolescent Diabetes (ISPAD) la misma debe realizarse al debut, anualmente en los primeros años y luego cada cinco años o cuando exista sospecha4.

La EC se asocia a otras inmunopatías como tiroiditis, hipoparatiroidismo ideopático, enfermedad de Addison y síndrome poliglandular autoinmune. Para la pesquisa de EC se solicitan Ac específicos como antitransglutaminasa, péptidos desaminados de gliadina o antiendomisio según experiencia y disponibilidad, y dosaje de IgA para descartar deficiencia y falsos resultados negativos. La confirmación diagnóstica se realiza por biopsia duodenal. Algunos autores sostienen que puede prescindirse de la misma si el título de anticuerpos es muy elevado (más de 10 veces el valor base) ${ }^{5}$.

En nuestra paciente es importante el seguimiento en cuanto a evolución y adherencia. Debe monitorearse el riesgo de aterosclerosis temprana y de complicaciones a largo plazo. Si bien no existen datos concluyentes, este grupo podría presentar un riesgo mayor favorecido por el HLA de base con una mayor tendencia a infecciones e inflamación $n^{6}$. Particularmente en esta paciente la obesidad funcionaría como una inflamación de bajo grado y un mayor riesgo adicionado de aterosclerosis.

\section{BIBLIOGRAFÍA}

1. Elfstrom P, Sundstrom J, Ludvigsson JF. Systematic review with meta-analysis: associations between coeliac disease and type 1 diabetes. Aliment PharmacolTher 2014 Nov; 40(10):1123-32.

2. Kahaly JG, Frommer L, Schuppan D. Celiac disease and endocrine autoimmunity: the genetic link. Autrev 2018. Doi:10.1016/j.autrev.2018.05.013.

3. Krishnareddy S. The microbiome in celiac disease. Gastroenterol Clin North Am 2019 Mar; 48(1):115-126. Doi: 10.1016/j. gtc.2018.09.008.

4. Pihoker C, Forsander G, Fantahun B, Virmani A, Corathers S, Benítez-Aguirre P, Fu J, Maahs DM. ISPAD Clinical Practice Consensus Guidelines 2018. The delivery of ambulatory diabetes care to children and adolescents with diabetes. Pediatr Diabetes 2018 Oct; 19 Suppl 27:84-104. Doi: 10.1111/pedi.12757. 
5. Husby S, Koletzko S, Korponay-Szabo IR, et al. European Society for Pediatric Gastroenterology, Hepatology, and Nutrition guidelines for the diagnosis of coeliacdisease. J Pediatr Gastroenterol Nutr 2012; 54:136-60.

6. Odermarsky M, Pesonen E, Sorsa T, Lernmark A, Pussinen PJ, Petru L. HLA, infections and inflammation in early stages of atherosclerosis in children with type 1 diabetes. Acta Diabetologica January 2018; Vol 55, Issue 1:41-47. Doi 10.1007/ s00592-017-1063-1.

\title{
SALÓN GRAN PANAMERICANO
}

\subsection{A $17.20 \mathrm{~h}$}

\section{CONFERENCIA: RELACIONES Y VÍNCULOS ENTRE PÁNCREAS EXOCRINO Y ENDOCRINO}

\author{
Disertante: Dr. Luis Grosembacher
}

Presidente: Dra. Solange Houssay

El páncreas endocrino y exocrino provienen del mismo botón embrionario y conviven en un mismo órgano: el páncreas. Sin embargo es habitual que en la práctica enfoquemos las enfermedades endocrinas, como diabetes mellitus, y las exocrinas, como pancreatitis, de una manera completamente independientes entre sí. No obstante, en la actualidad se identificaron numerosos mecanismos fisiológicos y fisiopatogénicos que relacionan el páncreas endocrino con el exocrino y que permiten alcanzar una mayor comprensión del vínculo que une a las enfermedades de ambos.

La diabetes causada por enfermedades del páncreas exocrino se denomina en la actualidad diabetes tipo 3c (DT3c). La pancreatitis crónica es la enfermedad pancreática más común como causa de DT3c. Otras pancreatopatías exocrinas, como el adenocarcinoma ductal, hemocromatosis, fibrosis quística y pancreatectomías parciales previas, también son causa menos frecuente de DT3c. En la pancreatitis crónica se identificaron algunos mecanismos patogénicos que conducen a la hiperglucemia. Uno de ellos es la expansión de la inflamación y fibrosis desde el territorio exocrino hacia los islotes pancreáticos lo cual provoca insulinopenia e hiperglucemia. Por otro lado, la hiperglucemia estimula a las llamadas "células estrelladas", responsables directas de inducir fibrosis en todo el tejido pancreático y también de apoptosis de células beta. De esta manera se establece un circuito que conduce a un daño progresivo y simultáneo, tanto en el tejido exocrino como endocrino.

Desde el punto de vista clínico, el paciente con DT3c presenta labilidad glucémica (por baja reserva insulínica y déficit de glucagón) y requiere insulinoterapia para su control. Además padece insuficiencia pancreática exocrina (IPE), secundaria a su pancreatopatía crónica, que se diagnostica con bajos niveles de elastasa en materia fecal y en este caso necesitaría terapia sustitutiva con enzimas digestivas.

Por otro lado, se observó una asociación no infrecuente de IPE en pacientes con diabetes mellitus tipo 1 ó tipo 2 (DM1 ó DM2). La prevalencia de la asociación es muy variable y oscila del 10 al 40\%. Mediante numerosos mecanismos la diabetes puede afectar el páncreas exocrino y provocar IPE. En el caso de DM1, la ausencia del efecto trófico de la insulina sobre las células acinares, la extensión de la destrucción autoinmune hacia los acinos y la estimulación de la hiperglucemia sobre las células estrelladas, son algunos mecanismos que contribuyen a la fibrosis pancreática. En pacientes con DM2, la neuropatía diabética autonómica, los fenómenos inflamatorios (macrófagos e interleuquinas), la pérdida de desmosomas (conexiones entre islotes y acinos) y la hiperglucemia, entre otros, son mecanismos patogénicos que conducen a la fibrosis pancreática.

En resumen, si una enfermedad compromete funcionalmente el páncreas endocrino o exocrino, con el tiempo inevitablemente también se comprometerá el otro sector pancreático.

\section{BIBLIOGRAFÍA}

- Hart P, Bellin MD, Andersen DK, et al. Consortium for the study of Chronic Pancreatitis, Diabetes and Pancreatic Cancer (CPDPC). Type 3c (pancreatogenic) diabetes mellitus secondary to chronic pancreatitis and pancreatic cáncer. Lancet Gastroenterol Hepatol 2016 Nov 1; (3): 226-237. 